\title{
Modelling space and modelling spatially: presentation of the special corner on "Spatial Models and Models of Space"
}

\author{
Thomas Hünefeldt
}

Published online: 12 January 2011

(C) Marta Olivetti Belardinelli and Springer-Verlag 2011

Following the 4th International Conference on Spatial Cognition (ICSC 2009), which had taken place in Rome in September 2009 (see http://w3.uniroma1.it/icsc/2009) and which had been devoted to "Spatial Cognition and Action" (see Cognitive Processing, vol. 10, Supplement 2), selected conference participants have been invited to contribute to a Special Corner on "Spatial Models and Models of Space". The selection was based on the quality of the research presented at the conference and on its relevancy with respect to the topic of the Special Corner, but contributors were left free to present research on problems different from those investigated in the research presented at the conference, if these problems matched the general topic of "Spatial Models and Models of Space". The six contributions published in the Special Corner passed the review process in time for this issue. Further invited contributions might be published informally in one of the following issues.

In ideating the topic of "Spatial Models and Models of Space", we had in mind two radically different, but at the same time closely related kinds of modelling. While the term "spatial models" was meant to refer to models that represent either spatial or non-spatial relationships in terms of spatial relationships, the term "models of space" was meant to refer to models that represent spatial relationships in terms of some either spatial or non-spatial relationships. Accordingly, there may be models that are both spatial models and models of space (e.g., geographical maps), but there may also be models that are spatial models but not models of space (e.g., diagrams of temporal or logical

T. Hünefeldt $(\square)$

Department of Psychology, University of Rome "La Sapienza",

Via dei Marsi, 78, I-00185 Rome, Italy

e-mail: thomas.huenefeldt@uniroma1.it relations) or that are models of space but not spatial models (e.g., symbolic representations of spatial relations). Furthermore, both spatial models and models of space may be object of higher-order models that may themselves be either spatial or non-spatial, so that there may be spatial models of non-spatial models of space (e.g., graphical representations of the meaning of symbolic representations of spatial relations) or non-spatial models of spatial models (e.g., algorithmic representations of diagrams). In the following, the contributions to the Special Corner will be localized within this taxonomy of first- and second-order spatial models and models of space, and their research questions will be specified on this basis.

Most contributions to this Special Corner are in some way concerned with the construction and evaluation of spatial models or models of space. The first contribution, "Visualizing Space, Time, and Agents: Production, Performance, and Preference" by Angela Kessell and Barbara Tversky, investigates subjects' production and use of spatial models that represent both spatial and non-spatial relationships. In particular, these authors examine the production and use of graphical visualizations aimed at keeping track of the different spatial locations of different agents at different times of the day. By contrast, the second contribution, "Multimodal Cognitive Interface for Robot Navigation" by Mohammed Mahfouz Elmogy, Christopher Habel and Jianwei Zhang, deals with both spatial and non-spatial models of spatial relationships. In fact, these researchers describe a robot navigation system with an interface that uses both verbal and graphical semi-formal route descriptions and evaluate subjects' interaction with this multimodal interface. The following two contributions are both concerned with the construction and evaluation of a particular kind of second-order spatial models of spatial relationships, i.e., spatial models representing the 
non-spatial models of spatial relationships that are implied in spatial language. While Mark M. Hall, Philip D. Smart and Christopher B. Jones, in their article "Interpreting Spatial Language in Image Captions", propose a system that allows for translating from the vague spatial language in image captions into the crisp representation required for modern Geographic Information Systems, John D. Kelleher, Robert J. Ross, Colm Sloan and Brian Mac Namee, in their article "The Effect of Occlusion on the Semantics of Projective Spatial Terms", investigate the hypothesis that perceptual factors, and in particular visual occlusion, affect the spatial templates of spatial propositions, and construct a mathematical model that better accounts for these effects than the adaptation of the well-established Attention Vector Sum model.

The remaining two contributions to the Special Corner do not involve the construction and evaluation of spatial models or models of space, but are focussed on the analysis of the cognitive functions involved in the use of such models. Thora Tenbrink and Inessa Seifert's article "Conceptual Layers and Strategies in Tour Planning" addresses the cognitive processes and strategies involved in a complex tour planning task that requires the integration of information from spatial and non-spatial models of space, namely from geographical maps, on the one hand, and space-related mental models and scripts, on the other. Finally, Peter E. Keller, Masami Ishihara and Wolfgang Prinz, in their article "Effects of Feedback from Active and Passive Body Parts on Spatial and Temporal Parameters in Sensorimotor Synchronization", question the homogeneity of the mental model of the pericorporeal space. In fact, these authors employ a sensorimotor synchronization paradigm to investigate how the timing and kinematics of the active finger are affected by tactile feedback from passive body parts that vary in sensitivity.

The topic of this latter contribution gives us the opportunity to direct the readers' attention to the next edition of the International Conference on Spatial Cognition, which will take place in Rome in September 2012 (see http://w3.uniroma1.it/icsc/2012/) and which will be devoted to "Space and Embodied Cognition". 